\title{
The Influence of Assisted Hopping Process on the Transport Properties of Quantum Dot with Ferromagnetic Leads
}

\begin{abstract}
G. GÓrski* And K. KuCAB
Faculty of Mathematics and Natural Sciences, University of Rzeszów, S. Pigonia 1, 35-310 Rzeszów, Poland

We study the transport properties of a quantum dot coupled to ferromagnetic leads with occupancy dependent hybridization. Using the modified equation of motion approach and modified perturbation theory we show that the assisted hopping processes can be the mechanism which is responsible for the generation of plateau in the linear conductance. This process breaks the particle-hole symmetry for conductance and magnetization. Moreover, the assisted hopping processes combined with the spin polarization cause the change of thermoelectric transport characteristics such as thermopower, the heat conductance and the figure of merit.
\end{abstract}

DOI: 10.12693/APhysPolA.135.85

PACS/topics: 72.10.Fk, 72.15.Qm, 73.63.Kv

\section{Introduction}

Actually, one of the widely discussed issues is the interplay between heat and charge flows in the quantum dot. The main effect for this interaction is the Seebeck effect in which the temperature gradient generates the thermovoltages. The discovery of the spin Seebeck effect [1] caused the increased interest in the spin-dependent transport in QD systems [2-12]. For the quantum dot system the spin dependent transport can be realized by the use of an external magnetic field [2-5] or ferromagnetic leads [6-12]. The spin current offers an opportunity for fast switching of the system's magnetization. This feature is one of the key technologies used today in spintronic devices.

Currently, there is a great interest in finding systems with high efficiency of the thermal to spin current conversion and the strong dependence of magnetization on carrier concentration. The use of assisted hopping process can have a great importance in such systems. The assisted hopping effect introduces the dependence of coupling between QD and metallic leads on the occupancy of the QD level by the electron with opposite spin, $V_{k \beta \sigma}\left(1-\alpha \hat{n}_{d-\sigma}\right)$. The $V_{k \beta \sigma}$ term describes the tunneling processes between the dot and electrodes where $\alpha$ is the assisted hopping parameter. The nearest-neighbor hopping integral, depending on the occupation of the opposite spin electrons (also known as correlated hopping), was used previously to describe the metallic ferromagnetism of the bulk systems (see e.g. [13] and the references cited therein). The correlated hopping causes the enhancement of magnetic ordering. There are two mechanisms used in the phenomena of ferromagnetic state increase. In the first one, the correlated hopping causes

\footnotetext{
*corresponding author; e-mail: ggorski@ur.edu.pl
}

spin-dependent band-shift and as a result it generates the effective exchange field (this is an equivalent of the Stoner mechanism). The second mechanism is based on the effective mass reduction, or equivalently, a bandwidth expansion, which leads to the spin-dependent density of states. As Hirsch has shown [14], the difference of majority and minority spin electrons bandwidth enhances the spin polarization. As a result, ferromagnetism arises from the combination of gains in potential and kinetic energy.

In this work, we study the effect of assisted hopping term on the spin-dependent thermoelectric transport characteristics of the quantum dot attached to ferromagnetic leads. The assisted hopping process in the quantum dot system was used in previous works to describe the coupling between quantum dot and external nonmagnetic leads (see e.g. [15]). The characteristic feature of assisted hopping is that this process breaks the electron-hole symmetry. This feature can explain the asymmetric dependence of QD conductance as a function of gate voltage, which is observed experimentally [3]. Extending the previous results [15] we take into account the coupling with ferromagnetic leads which creates an effective exchange field on the quantum dot $[8,12]$, which in turn causes the spin-dependence of transport coefficients. Additionally, the assisted hopping term enhances the magnetic moment of a quantum dot by adding the spin-dependent shift of quantum dot energy and by spindependent coupling of quantum dot and ferromagnetic leads. The combination of assisted hopping term with the leads' polarization effect allows us to obtain the system characterized by the strong dependence on spin direction. The system proposed by us is described by the extended single impurity Anderson model, where we use the alternative equation of motion approach [16]. This approach for intermediate values of the Coulomb repulsion $(U \approx 5 \Gamma)$ is comparable with the numerical normalization group and Quantum Monte Carlo calculations [16]. 


\section{The model}

The considered system contains a single-level quantum dot attached to two ferromagnetic electrodes. We assume that the coupling between the quantum dot and ferromagnetic electrodes will depend on the QD occupation. The Hamiltonian of our model has the form

$$
\begin{aligned}
H & =\sum_{\sigma} \varepsilon_{d \sigma} \hat{n}_{d \sigma}+U \hat{n}_{d \uparrow} \hat{n}_{d \downarrow}+\sum_{\substack{k \sigma \\
\beta=L, R}}\left(\varepsilon_{k \beta}-\mu_{\beta}\right) \hat{n}_{k \beta \sigma} \\
& +\sum_{\substack{k \sigma \\
\beta=L, R}}\left[V_{k \beta \sigma}\left(1-\alpha \hat{n}_{d-\sigma}\right) d_{\sigma}^{+} c_{k \beta \sigma}+\text { h.c. }\right]
\end{aligned}
$$

where $d_{\sigma}^{+}\left(d_{\sigma}\right)$ are the creation (annihilation) operators for the dot electron with spin $\sigma, c_{k \beta \sigma}^{+}\left(c_{k \beta \sigma}\right)$ are the creation (annihilation) operators for the ferromagnetic lead electron, $\beta=L, R$ corresponds to the left and right leads, respectively, $\varepsilon_{k \beta}$ is the energy dispersion of $\beta$ lead, $\mu_{\beta}$ is the chemical potential of $\beta$ lead, $U$ is the on-site Coulomb interaction between electrons on the dot, and $\varepsilon_{d \sigma}$ is the dot energy.

The hopping matrix element $V_{k \beta \sigma}$ describes the tunneling processes between the dot and ferromagnetic electrodes and $\alpha$ is the assisted hopping parameter. The parallel or antiparallel magnetization of left and right leads is possible. Because the antiparallel magnetization of leads gives the similar results as non-magnetic leads [6, 12], we will focus on the parallel orientation of the leads' magnetizations. In our model the effective coupling of quantum dot to the spin $\sigma$ band of the ferromagnetic lead, $\Gamma_{\sigma}^{\mathrm{eff}}$, is dependent on the assisted hopping parameter $\alpha$ and on the occupation of the quantum dot by electron with opposite spin. Assuming that the left and right lead are identical and using flat density of states in the leads we obtain the following expression:

$$
\Gamma_{\sigma}^{\mathrm{eff}}=\left(1-\alpha n_{d-\sigma}\right)^{2} \Gamma(1+\sigma p),
$$

where $p$ is the spin polarization of the lead.

The spin polarization of the lead changes the value of effective coupling and additionally creates an effective exchange field on the quantum dot $[8,12]$. The additional spin-dependent shift of quantum dot energy is obtained via the correlation parameter $B_{\sigma}$, which can be written as [13]:

$$
B_{\sigma}=\frac{2 \alpha}{1-\alpha n_{d-\sigma}} \int_{-\infty}^{\infty} \frac{1}{\pi} \operatorname{Im}\left(\Gamma_{\sigma}^{\mathrm{eff}} G_{d \sigma}(\omega)\right) f(\omega) \mathrm{d} \omega,
$$

where $G_{d \sigma}(\omega)$ is the quantum dot Green function. To obtain this function we employ the alternative equation of motion method $[13,16]$. As the result we obtain

$$
G_{d \sigma}(\omega)=\frac{1}{\omega-\varepsilon_{d \sigma}-\mathrm{i} \Gamma_{\sigma}^{\mathrm{eff}}-\Sigma_{d \sigma}(\omega)},
$$

where the quantum dot self-energy is given by

$$
\Sigma_{d \sigma}(\omega)=U n_{d-\sigma}+B_{\sigma}+\Sigma_{d \sigma}^{\prime}(\omega)
$$

and $\Sigma_{d \sigma}^{\prime}(\omega)=\Sigma_{d \sigma}^{(2)}(\omega) /\left(1+A_{1} \Sigma_{d \sigma}^{(2)}(\omega)\right)$ is the higher order part of self-energy [16].

\section{Numerical results}

Electron transport through the quantum dot system is accompanied with the transfer of both charge and heat. In the linear temperature and bias regime, the charge current and the heat current can be expressed by the help of transport coefficients: the spin-dependent electrical conductance

$$
G_{0 \sigma}=e^{2} I_{0 \sigma}
$$

and the spin-dependent thermoelectric power

$$
S_{\sigma}=-\frac{1}{2|e| T} \frac{I_{1 \sigma}}{I_{0 \sigma}},
$$

where the thermal integrals are defined as:

$$
I_{n \sigma}=\frac{1}{h} \frac{4 \pi \Gamma_{\sigma L}^{\mathrm{eff}} \Gamma_{\sigma R}^{\mathrm{eff}}}{\Gamma_{\sigma}^{\mathrm{eff}}} \int_{-\infty}^{\infty}(\omega-\mu)^{n}\left(-\frac{\partial f}{\partial \omega}\right) \rho_{d \sigma}(\omega) \mathrm{d} \omega .
$$

In Fig. 1 we present the dependence of linear conductance $G_{0}=G_{0 \uparrow}+G_{0 \downarrow}$ (Fig. 1a) and spin-polarization of linear conductance $P_{G}=\left(G_{0 \uparrow}-G_{0 \downarrow}\right) /\left(G_{0 \uparrow}-G_{0 \downarrow}\right)$ (Fig. 1b) on the dot energy for several values of leads' spin polarization $p$. In the computations we use the assisted hopping parameter $\alpha=0.2, T=0.1 \Gamma$ and $U=5 \Gamma$. Using the non-zero value of $\alpha$ parameter causes the asymmetric character of obtained transport coefficients. In Fig. 1a we show the $G_{0}\left(\varepsilon_{d}\right)$ dependence for several values of $p$. For $p=0$ we observe the conductance plateau, which is also observed experimentally (see e.g. [3]).

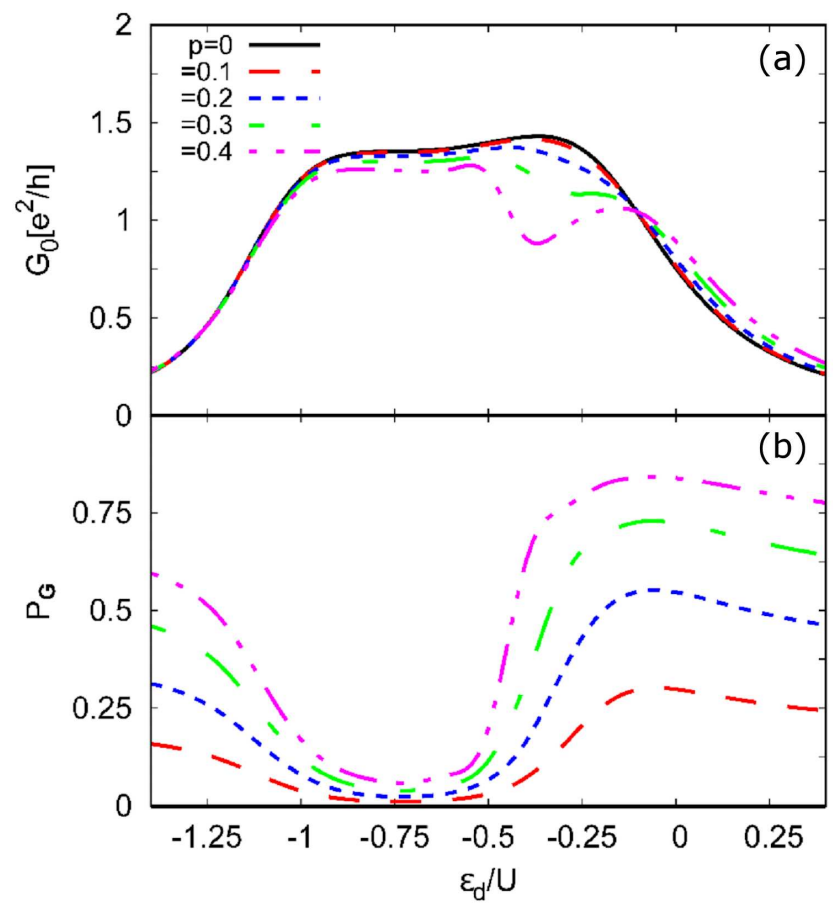

Fig. 1. The linear conductance (a) and the spin polarization of conductance (b) as a function of the dot energy for different values of the polarization of leads. The other parameters are: $U=5 \Gamma, T=0.1 \Gamma$ and $\alpha=0.2$. 
The increase of leads' spin polarization generates the effective exchange field, which brings to the reduction of the linear conductance [4]. The especially strong reduction occurs for the $\varepsilon_{d}>-U / 2$ region, i.e. in the region in which the effective exchange field is additionally enhanced by the correlation parameter $B_{\sigma}$. For $\alpha=0$ we obtain the symmetric character of $G_{0}\left(\varepsilon_{d}\right)$ (see e.g. [6, 7]). The effective exchange field, created by spin polarization of the leads, is enhanced by the correlation parameter $B_{\sigma}$, which leads to stronger split of QD spectral function. If we will use additionally the external magnetic field, we can obtain the spin polarization of linear conductance effect, without the Kondo resonance splitting (see [12]).

In Fig. 1b we show the dependence of spin polarization of linear conductance, $P_{G}\left(\varepsilon_{d}\right)$, on the dot energy. The value of $P_{G}\left(\varepsilon_{d}\right)$ is positive, so $G_{0 \uparrow}>G_{0 \downarrow}$, which proves that the tunneling of spin-up electrons compared to spindown electrons dominates. For $\alpha=0$ the dependence of $P_{G}\left(\varepsilon_{d}\right)$ has the minimum for particle-hole symmetry point $\varepsilon_{d}=-U / 2$. The non-zero value of $\alpha$ parameter shifts the minimum of $P_{G}\left(\varepsilon_{d}\right)$ towards $\varepsilon_{d} \approx-0.75 U$. For $\varepsilon_{d}>-U / 2$ region, the spin polarization of linear conductance rapidly grows.

In Fig. 2a we show the dependence of thermopower $S=$ $S_{\uparrow}+S_{\downarrow}$ on the quantum dot's energy. The thermopower changes its $\operatorname{sign}$ near $\varepsilon_{d} \approx-0.75 U$. At $\varepsilon_{d} \approx-1.1 U$ $\left(\varepsilon_{d} \approx-0.1 U\right)$ we observe the local maximum (minimum) of $S$. Using the non-zero value of $\alpha$ parameter causes the

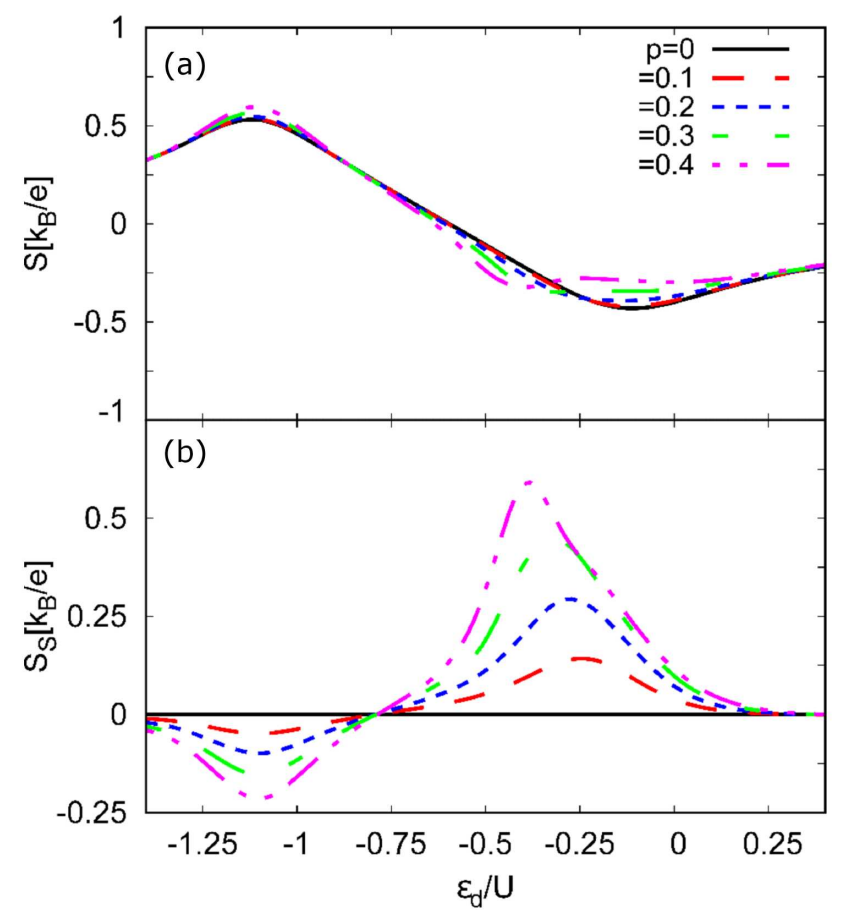

Fig. 2. The thermopower (a) and spin thermopower (b) as a function of the dot energy for different values of polarization of the leads. The other parameters are: $U=5 \Gamma, T=0.1 \Gamma$ and $\alpha=0.2$. disturbance of antisymmetric character of $S\left(\varepsilon_{d}\right)$ dependence. Higher values of $S$ are achieved for $\varepsilon_{d}<-0.75 U$, because when one decrease $\varepsilon_{d}$, the $T / \Gamma^{\mathrm{eff}}$ ratio increases, which leads to the increase of thermopower [7]. The increase of electrode polarization causes the additional enhancing of $S$ for $-1.25 U<\varepsilon_{d}<-0.75 U$, and weakening of $S$ for $-0.75 U<\varepsilon_{d}<0.25 U$. Additionally, for $-0.75 U<\varepsilon_{d}<0.25 U$ one can observe that the thermopower minimum shifts towards lower dot's energy. The polarization growth of the ferromagnetic leads causes the weak increase of total thermopower, whereas the thermopower associated with the spin up (down) electrons changes radically. As Krawiec and Wysokiński [10] showed, in the model where $U=\infty$ the increase of polarization increases the value of $S_{\uparrow}$ and additionally decreases the value of $S_{\downarrow}$. The spin dependence of thermopower can be expressed by the $S_{S}=S_{\uparrow}-S_{\downarrow}$ factor. For the finite values of $U$ the difference $S_{\uparrow}-S_{\downarrow}$ will be dependent on the dot energy (see [7]). The spin thermopower as a function of the dot energy for different spin polarization $p$ is shown in Fig. 2b. For zero spin polarization the spin thermopower vanishes. The increased value of leads' spin polarization causes the noticeable increase of $S_{S}$ for $-0.75 U<\varepsilon_{d}<0.25 U$, i.e. in the area for which the polarization of the quantum dot is strong. As in the case of $S$, there is the shift of the maximum of $S_{S}$ value towards lower dot's energies. For $\varepsilon_{d}<-0.75 U$, where the polarization of QD is much weaker, we obtain much less change of $S_{S}$ and we observe that the position of $S_{S}$ minimum value does not change.

\section{Conclusions}

In this paper we analyzed the spin dependence of the electrical conductance and the thermopower of quantum dot coupled to ferromagnetic leads. In the considered model we have taken into account the influence of assisted hopping process on the thermoelectric transport properties. The calculations were performed using the modified equation of motion approach for extended single impurity Anderson model. The numerical results showed that the assisted hopping process breaks the particle-hole symmetry of both the zero-bias differential conductance and the thermopower. This process is one of the methods to obtain the asymmetry of conductance and the thermopower. Another way of obtaining the transport coefficients asymmetry is to consider an energy- and spin-dependent band structure of the leads (e.g. a square-root shape DOS or parabolic band with some additional spin asymmetry as shown in [11]). The assisted hopping process can be the mechanism which is responsible for the linear conductance plateau generation. For the non-zero values of electrode polarization this process enhances the effective exchange field for $-0.75 U<\varepsilon_{d}<0.25 U$, causing the strong increase of the spin polarization of linear conductance and the spin thermopower. For $\varepsilon_{d}<-0.75 U$ the influence of assisted hopping parameter on the transport properties is much weaker. 


\section{Acknowledgments}

This work has received support from Faculty of Mathematics and Natural Sciences, Rzeszów University within the project No. WMP/GD-06/2017, and partial support from Center for Innovation and Transfer of Natural Sciences and Engineering Knowledge at the Rzeszów University.

\section{References}

[1] K. Uchida, S. Takahashi, K. Harii, J. Ieda, W. Koshibae, K. Ando, S. Maekawa, E. Saitoh, $\mathrm{Na}$ ture (London) 455, 778 (2008).

[2] T. Rejec, R. Žitko, J. Mravlje, A. Ramšak, Phys. Rev. B 85, 085117 (2012).

[3] A.V. Kretinin, H. Shtrikman, D. Goldhaber-Gordon, M. Hanl, A. Weichselbaum, J. von Delft, T. Costi, D. Mahalu, Phys. Rev. B 84, 245316 (2011).

[4] A.A. Aligia, Phys. Rev. B 74, 155125 (2006).

[5] R. López, D. Sánchez, Phys. Rev. Lett. 90, 116602 (2003).

[6] I. Weymann, Phys. Rev. B 83, 113306 (2011).
[7] I. Weymann, J. Barnaś, Phys. Rev. B 88, 085313 (2013).

[8] J. Martinek, M. Sindel, L. Borda, J. Barnaś, J. König, G. Schön, J. von Delft, Phys. Rev. Lett. 91, 247202 (2003).

[9] W. Rudziński, J. Barnaś, R. Świrkowicz, M. Wilczyński, Phys. Rev. B 71, 205307 (2005).

[10] M. Krawiec, K.I. Wysokiński, Phys. Rev. B $\mathbf{7 3}$ 075307 (2006).

[11] M. Sindel, L. Borda, J. Martinek, R. Bulla, J. König, G. Schön, S. Maekawa, J. von Delft, Phys. Rev. B 76, 045321 (2007).

[12] J. Martinek, Y. Utsumi, H. Imamura, J. Barnaś, S. Maekawa, J. König, G. Schön, Phys. Rev. Lett. 91, 127203 (2003).

[13] G. Górski, J. Mizia, K. Kucab, Phys. Status Solidi B 253, 1202 (2016).

[14] J.E. Hirsch, Phys. Rev. B. 59, 6256 (1999).

[15] S.B. Tooski, A. Ramšak, B.R. Bułka, R. Žitko, New. J. Phys. 16, 055001 (2014).

[16] G. Górski, J. Mizia, K. Kucab, Physica E 73, 76 (2015). 